\title{
MONITORING THE MICROARCSECOND QUASAR J1819+3845
}

\author{
J. DENNETT-THORPE and A.G. DE BRUYN \\ Kapteyn Institute, Groningen; ASTRON, Dwingeloo, The Netherlands
}

\begin{abstract}
We present the results of a year-long monitoring campaign on J1819+3845. We interpret the results of this WSRT campaign to infer critical source parameters such as source lifetime and structure on tens of microarcseconds. The long lifetime of the source at such high brightness temperatures requires continuous energy injection or exotic emission processes. We have previously interpreted the extreme scintillation of J1819+3845 as due to a relatively nearby $(\sim 20 \mathrm{pc})$ scattering screen. We show this scrcen has a velocity w.r.t. the LSR of $\sim 25 \mathrm{~km} \mathrm{~s}^{-1}$, as measured by the changing scintillation properties throughout the year: the 'velocity parallax'.
\end{abstract}

\section{Introduction}

In May 1999 we obtained observations of the quasar J1819+3845 $(z=0.54)$ that showed it to be the most extremely variable source known in the radio sky (Dennett-Thorpe and De Bruyn, 2000). The variations occur on timescales less than an hour and showed over $300 \%$ peak to peak variations.

A source exhibiting such variations due to intrinsic changes would have to be so small that the source would be expected to scintillate. We therefore interpreted the variations as solely due to refractive interstellar scintillation in the ISM (see Narayan, 1992) and references therein), and found good agreement with theory, with maximum variations occurring around $5 \mathrm{GHz}$, as the scintillation changed from the strong to weak regimes (Walker, 1998).

Interpreted in this manner, using standard scintillation theory, we conclude that the source is less than $30 \mu$ arcsecs in diameter at $5 \mathrm{GHz}$. This corresponds to 5 light-months, and (with at least $55 \%$ of the source's flux density in this small scintillating component) requires a brightness temperature $T_{B}>510^{12} \mathrm{~K}$. The scattering is found to be very close by $(\sim 20 \mathrm{pc})$, and may be associated with the edge of the Local Bubble (see Bhat et al., 1999).

The high brightness temperature of the source is particularly interesting in light of the Compton limit at $\sim 510^{11} \mathrm{~K}$ : sources can only exceed this limit in a transient manner, by highly relativistic outflow, or by exotic (coherent) emission mechanisms. We therefore launched a monitoring campaign to address the longevity of the source (the scintillating after a number of weeks (Kedziora-Chudczer et al., 1997). In addition, if the source was to continue to exhibit scintillation throughout the year, we could use this data to address both the source structure (potentially achieving 


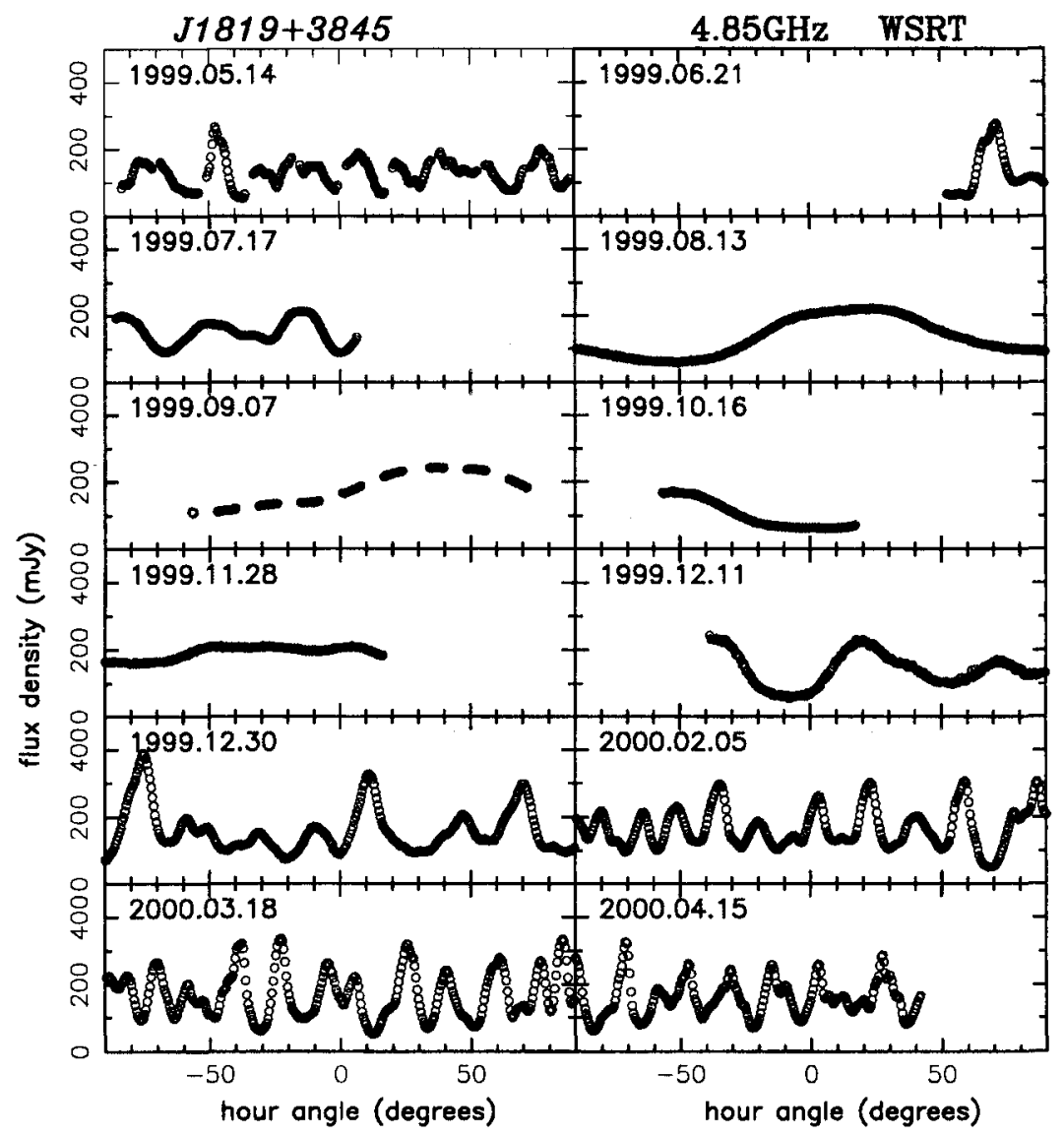

Figure 1. Selected observations over the period May 1999-May 2000. Each plot spans 12 hrs. WSRT $5 \mathrm{GHz} .120 \mathrm{~s}$ averaging time.

an 'image resolution' of tens of microarcseconds) and the velocity of the scattering plasma (a critical unknown in the determination of the source parameters).

We conducted this campaign at $5 \mathrm{GHz}$, where the scintillation was strongest, using the WRST. We observed the source for 12 hours each a month, with the observations spread over one or two sessions.

\section{Results and Interpretation}

Over the course of the year, the speed of the modulations slowed down in July through October, before speeding up once again by December. Figure 1 illustrates this with selected observations. The depth of the modulations appeared unchanged throughout the year. 


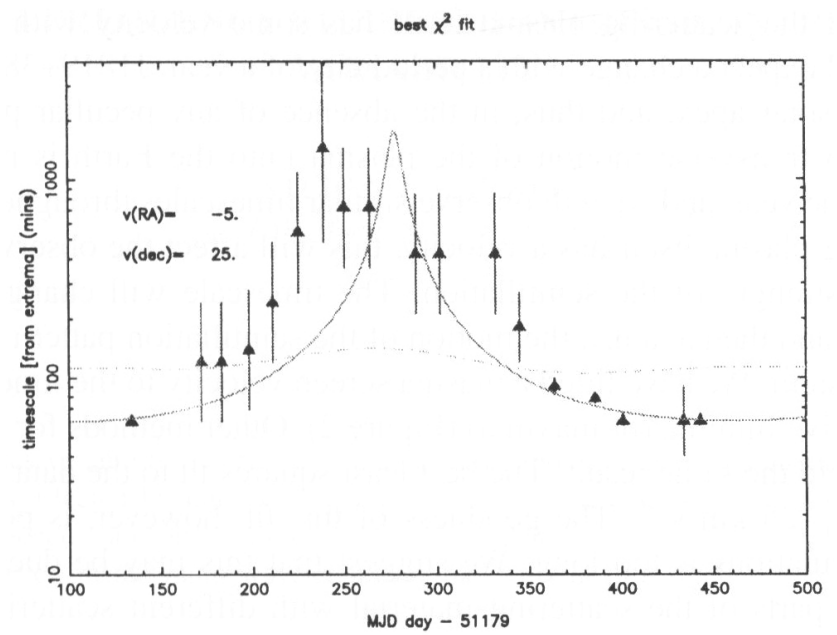

Figure 2. The timescales of the scintillations throughout the year. The solid line corresponds to best $x^{2}$ fit for a velocity of the scattering plasma, the dotted line is the prediction if the scattering plasma at rest in the LSR. Error bars are plotted on all observations.

There are four possible causes for changes in the observed scintillation characteristics. They are: change in the scattering plasma; a change in the source itself over this period; source structure; and a peculiar velocity of the scattering plasma.

A change in the scattering plasma, potentially either in our line of sight, or due to motions in the medium, is unlikely. For reasonable ISM velocities (tens of $\mathrm{km}$ $\mathrm{s}^{-1}$ ), a scattering medium no more than a few AU thick would be implied. This would require infeasibly high electron densities and turbulence.

An expansion of the source will increase the timescale $\tau$ of the modulations (as $\tau \propto R$ for strong scattering, where $R$ is the linear source size), as well as reduce the depth of the modulations (the normalised $r m s$ fluctuations, or the modulation index $m \propto R^{-7 / 6}$ ). There is no evidence that the modulation index has dropped in the period between July and mid-December: there are still excursions to $60 \mathrm{mJy}$ and $250 \mathrm{mJy}$. Furthermore, any changes in flux density, as might be expected to accompany such a change, appear minimal.

If the source showed structure, the scintillation pattern would be non-isotropic, and we would sample different directions through this during the year. In particular, if the source was elongated, with the smaller axis $\lesssim$ the Fresnel scale, then at a given time the Earth would be moving perpendicular to the source, and 3 months later, parallel to it. This would cause a change in the observed scintillation timescale and modulation index. Such a mechanism does not cause the observed changes because (i) once again this predicts an anti-correlation between $m$ and $\tau$ (ii) the period of the observed changes is 6 months, whilst our observations are much better fitted with a period of one year. 
Finally, if the scattering plasma itself has some velocity with respect to the LSR, we will expect a change with a periodicity of a year. J1819+3845 is observed close to the solar apex, and thus, in the absence of any peculiar plasma motion, the projected transverse motion of the plasma onto the Earth is nearly constant throughout the year, and we will observe similar timescales throughout this time. If the scattering plasma itself has a velocity, this will affect the observed timescales, but not the strength of the scintillation. The timescale will change as the Earth moves with, and then against, the motion of the scintillation pattern projected onto the Solar System. We have fitted a plasma screen velocity to the timescales derived from successive minima (or maxima) (Figure 2). Other methods for calculating the timescale yield the same result. The best least squares fit to the data gives a plasma velocity of $\sim 25 \mathrm{~km} \mathrm{~s}^{-1}$. The goodness of this fit, however, is poor: the period of slow modulations is too long. We suggest that this may be due to differential velocities in parts of the scattering material with different scattering strengths although the details of this have yet to be worked out.

\section{Conclusions}

(i) The source has remained with $55 \%$ of its flux density within a diameter $<$ $30 \mu$ arcsec at $5 \mathrm{GHz}$ for over a year.

(ii) The maintenance of an apparent brightness temperature of $>510^{12} \mathrm{~K}$ (at $5 \mathrm{GHz}$ ) for this period requires a continuous energy input.

(iii) The scattering plasma has a velocity w.r.t. the LSR of around $25 \mathrm{~km} \mathrm{~s}^{-1}$. This supports the notion that the scattering does not occur uniformly throughout the Galaxy, but in a smaller localised structure.

\section{References}

Bhat, N.D.R., Gupta, Y. and Rao, A.P.: 1998, Astrophys. J. 500, 262.

Dennett-Thorpe, J. and de Bruyn, A.G.: 2000, Astrophys. J. 529, L65.

Kedziora-Chudczer, L., Jauncey, D.L., Wieringa, M.H., et al.: 1997, Astrophys. J. 490, L9.

Narayan: 1992, Phil. Trans. R. Soc. Lond. 341, 151.

Walker, M.A.: 1998, Mon. Not. R. Astron. Soc. 294, 307. 